\title{
Controlling the Fano interference in a plasmonic lattice
}

\author{
A. Christ, ${ }^{1, *}$ Y. Ekinci, ${ }^{2}$ H. H. Solak,${ }^{3}$ N. A. Gippius, ${ }^{4,5}$ S. G. Tikhodeev,${ }^{5}$ and O. J. F. Martin ${ }^{1}$ \\ ${ }^{1}$ Nanophotonics and Metrology Laboratory, Swiss Federal Institute of Technology Lausanne, 1015 Lausanne, Switzerland \\ ${ }^{2}$ Laboratory of Metal Physics and Technology, Swiss Federal Institute of Technology Zürich, 8093 Zürich, Switzerland \\ ${ }^{3}$ Laboratory for Micro- and Nanotechnology, Paul Scherrer Institut, 5232 Villigen, Switzerland \\ ${ }^{4}$ LASMEA, UMR 6602 CNRS, Université Blaise Pascal, 63177 Aubière, France \\ ${ }^{5}$ A. M. Prokhorov General Physics Institute RAS, Moscow 119991, Russia
}

(Received 6 September 2007; published 16 November 2007)

\begin{abstract}
We analyze the influence of near-field coupling on the formation of collective plasmon modes in a multilayer metallic nanowire array. It is shown that the spectral interference between super- and subradiant normal modes results in characteristic line shape modifications which are directly controlled by the spacing as well as the alignment of the stacked lattice planes. Moreover, a distinct near-field-induced reversal of particle plasmon hybridization is reported. Our numerical findings are in excellent agreement with experimental results.
\end{abstract}

DOI: $10.1103 /$ PhysRevB.76.201405

PACS number(s): 42.70.Qs, 61.46.-w, 73.20.Mf

\section{INTRODUCTION}

Radiative decay phenomena in atomic or solid state systems have attracted a lot of interest in recent years. For example, it is well known that light-mediated coupling in an ensemble of resonant two-level systems results in the formation of collective states with cooperative radiative decay characteristics. ${ }^{1}$ In particular, super- or subradiant damping can be observed, depending on the symmetry of the induced normal modes. Similar to studies on optical lattices, ${ }^{2}$ radiative interwell coupling in Bragg-periodic multiple quantum well structures, i.e., resonant one-dimensional photonic crystals, has been investigated as an idealized model system.,4

Layered metallic nanostructures supporting localized surface plasmon polariton modes ${ }^{5}$ (i.e., so-called particle plasmons) are excellent candidates to investigate comparable interlayer coupling phenomena in an alternative material system. Especially with regard to future applications, ${ }^{6}$ the control over radiative coupling will allow the design of novel plasmonic materials with a tailored optical response. ${ }^{7}$ The simplest approach to study collective plasmonic excitations in a layered geometry considers stacked nanowire arrays which form vertically aligned wire pairs. Such structures have recently gained a lot of attention due to their capability to mimic magnetic atoms and to modify the magnetic permeability at infrared or optical wavelengths. ${ }^{8-11}$

Fundamental properties of resonant metallic nanostructures, such as localized enhanced fields and the efficient conversion of propagating light, ${ }^{12}$ strongly differentiate them from their atomic or solid state counterparts. In particular, the radiative damping of localized surface plasmon modes is generally characterized by decay times in the femtosecond range, which effectively limits the coherent interaction length. Moreover, the presence of strong near-field components due to particle plasmon excitations affects the radiative interlayer coupling in an ensemble of closely spaced wire pairs. Hence, the question arises how the formation of collective plasmonic eigenmodes is influenced (e.g., lifetime and energy of the normal modes) by adjusting the near-field interaction.

In this Rapid Communication, we study coherent interlayer coupling in a plasmonic lattice. It is discussed how particle plasmon hybridization ${ }^{13}$ and the spectral interference between super- and subradiant plasmonic eigenmodes are controlled by tuning the geometrical properties of the lattice, i.e., by modifying the effect of near-field interaction.

\section{SAMPLE AND THEORETICAL DESCRIPTION}

As schematically displayed in Fig. 1, the lattice structure consists of two aligned and vertically spaced gold nanowire arrays. The arrays are characterized by an identical period $d_{x}$ and are displaced horizontally by $d_{s}$. The propagation direction of the incident light field is along the $z$ axis, i.e., perpendicular to the grating planes. Only $p$ polarization (magnetic field parallel to the nanowires) is considered, since no plasmonic features are expected for $s$ polarization. All numerical calculations are based on a scattering-matrix formalism. ${ }^{14}$ The method has already proven its capability to handle metallic wire structures. ${ }^{15,16}$ Only the geometrical properties of the layered structure and the dielectric susceptibilities of gold $^{17}$ and silica $\left(\varepsilon_{s}=2.13\right)$ are taken into account. Note that the essential physics is first discussed theoretically on example of an idealized structure embedded into a dielectric (silica) matrix. Our theoretical analysis is then experimentally checked for two characteristic cases.

\section{NUMERICAL RESULTS}

In Fig. 2, the tunability of the optical response is illustrated by a first series of spectra. Numerically calculated re-

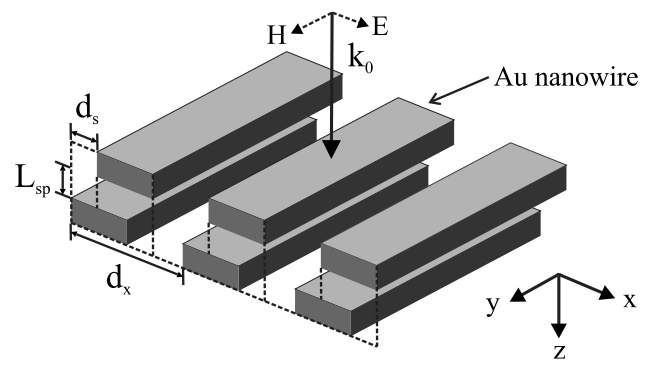
lattice.

FIG. 1. Schematic view of the double-layer plasmonic 

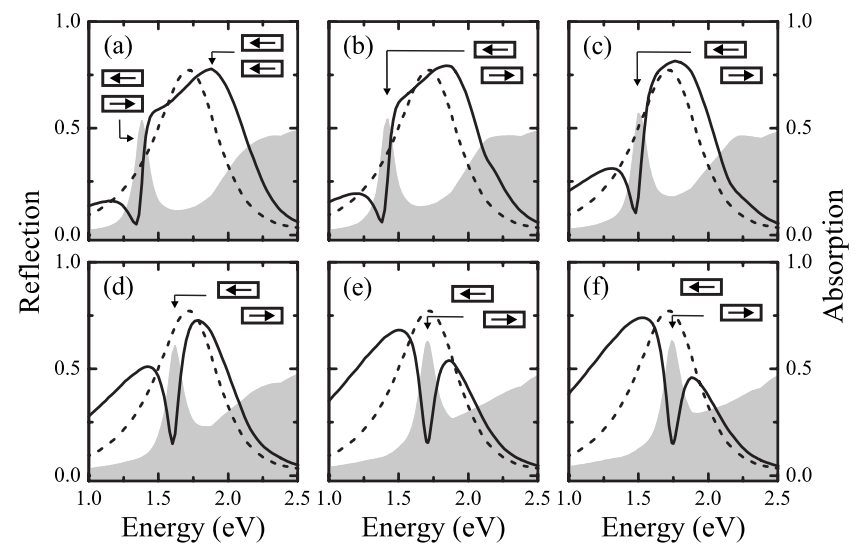

FIG. 2. Reflection (solid lines) and absorption spectra (shaded areas) for structures with $d_{x}=200 \mathrm{~nm}$ and $L_{s p}=30 \mathrm{~nm}$ (normal incidence, $p$ polarization). The lateral shift is increased from $d_{s}=0$ (a) to $100 \mathrm{~nm}$ (f) in steps of $20 \mathrm{~nm}$. The reflection spectra of a single grating layer (dashed lines) are shown as reference.

flection and absorption spectra are plotted for plasmonic lattices with a constant period of $d_{x}=200 \mathrm{~nm}$, a nanowire cross section of $100 \times 15 \mathrm{~nm}^{2}$, and a spacer thickness of $L_{s p}$ $=30 \mathrm{~nm}$. Only the lateral displacement $d_{s}$ is modified and stepwise increased. For reasons of simplicity, the lattice structure is embedded in a homogeneous silica matrix. The additionally plotted spectra of a single reference lattice are characterized by one spectrally broad particle plasmon resonance, corresponding to the optically active symmetric eigenmode of the lattice (in-phase oscillation of the valence electrons in all wires, dashed lines in Fig. 2). The period is chosen such that near-field interaction between adjacent wires is minimized and only the zeroth-order diffraction channels are propagating. The spectral response clearly changes when a second identical grating layer is placed within a few nanometers above the first layer. The plasmonic

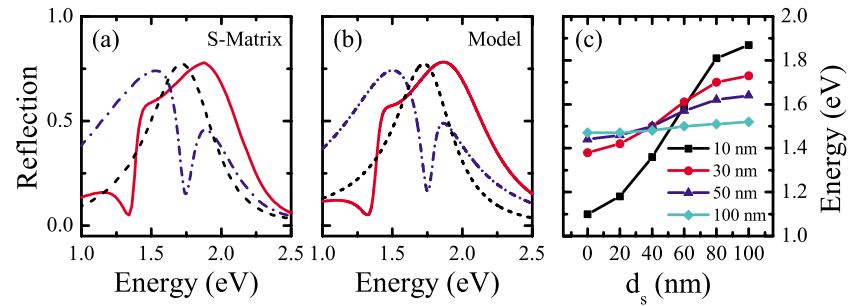

FIG. 3. (Color online) Numerically obtained reflection spectra for structures with $L_{s p}=30 \mathrm{~nm}$ (a) are compared with model simulations (b). Spectra are shown for $d_{s}=0$ (solid red lines), $100 \mathrm{~nm}$ (dash-dotted blue lines), and the single-layer reference (dashed black lines). (c) indicates the calculated shift of the antisymmetric eigenmode for spacer thicknesses of 10, 30, 50, and $100 \mathrm{~nm}$.

double-layer structure exhibits two pronounced resonances (i.e., lifted degeneracy) due to the interaction of the plasmon modes of the individual lattices. Figure 2(a) resembles the prominent case of wire pair structures. ${ }^{8,9}$ The high-energy resonance is associated with the symmetric or superradiant eigenmode (i.e., electric resonance). As indicated by the inset, the impinging light induces a parallel electron flow in the wire pair. The antisymmetric or subradiant eigenmode (i.e., magnetic resonance) is found at a lower energy and is characterized by opposing currents. The subradiant nature of the mode, i.e., the suppression of radiative damping, results in a prolonged lifetime (narrow spectral bandwidth) and a pronounced absorption peak. Note that the spectral overlap of the long-living subradiant mode and the spectrally broad superradiant mode leads to the typical Fano-type interference, ${ }^{18}$ inducing asymmetric line shapes in the reflection spectrum. In Fig. 2, the tunability of the optical response becomes evident when increasing the lattice detuning $d_{s}$. The modified near-field interaction changes the eigenmode properties and hence the surface plasmon interference conditions. The most striking feature is the strong blueshift of the antisymmetric
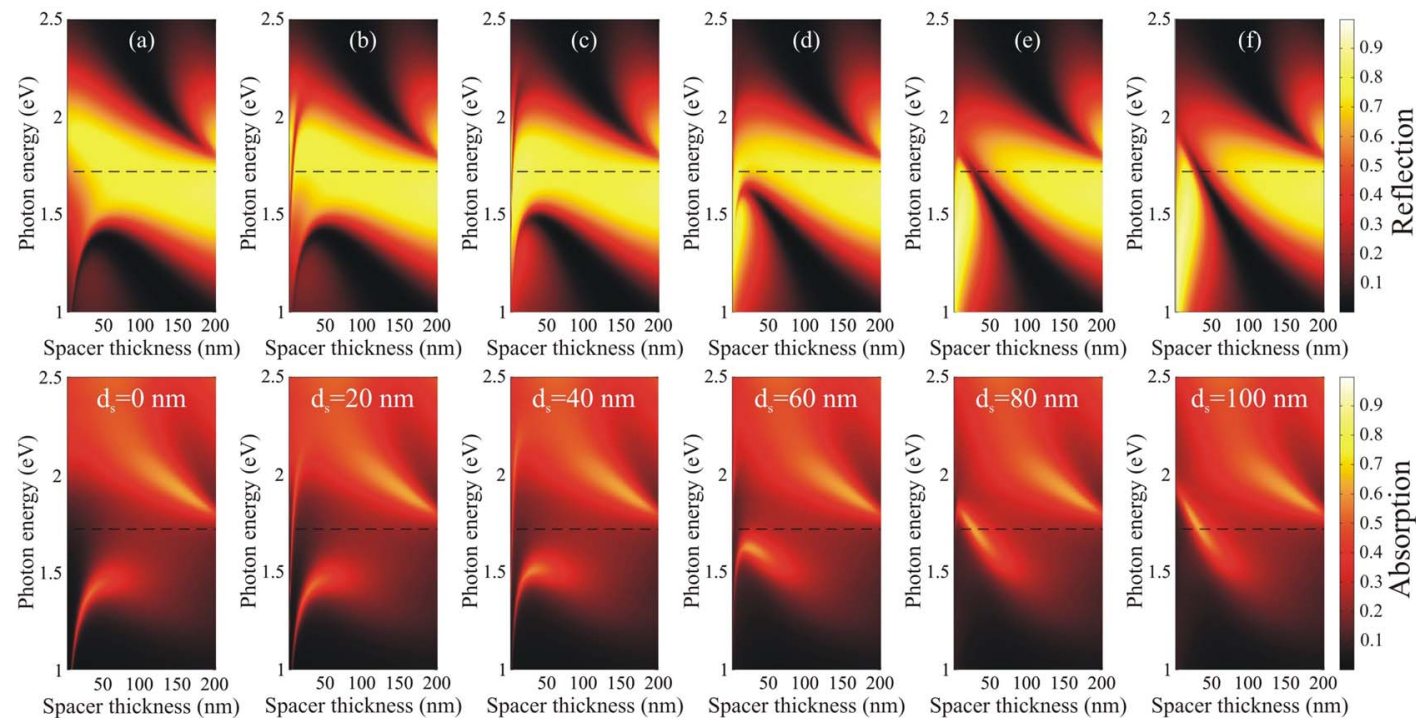

FIG. 4. (Color online) Calculated reflection (upper row) and absorption (lower row) contour maps for lattice structures with $d_{x}$ $=200 \mathrm{~nm}$ plotted as a function of $L_{s p}$. The lateral shift is increased from $d_{s}=0$ (a) to $100 \mathrm{~nm}$ (f) in steps of $20 \mathrm{~nm}$. The spectral position of the localized plasmon mode of a single grating layer is indicated by dashed horizontal lines. 
eigenmode, which shows up as a pronounced reflection minimum within the symmetric surface plasmon resonance.

The Fano-type line shapes in the far-field reflection spec$\operatorname{tra} R(\omega)=|r(\omega)|^{2}$ can be well described by a phenomenological model ${ }^{7,19,20}$ assuming

$$
r(\omega)=a_{r}+\sum_{j=s, a} \frac{-b_{j} \Gamma_{j} e^{i \phi_{j}}}{\hbar \omega-E_{j}+i \Gamma_{j}},
$$

where $a_{r}$ specifies the amplitude of a constant background, and $b_{j}$ and $\phi_{j}$ characterize the amplitude and phase of the two localized surface plasmon eigenmodes. The spectral position and the linewidth of the individual resonances are specified by $E_{j}$ and $\Gamma_{j}$, respectively. Figure 3 shows numerical calculated reflection spectra [Fig. 3(a)] and the corresponding fits [Fig. 3(b)] using Eq. (1) for three characteristic cases extracted from Fig. 2. For the single-layer reference spectrum, the fit includes only a single resonance at $E_{s}$ $=1.75 \mathrm{eV}$ with a total linewidth $\Gamma_{s}=250 \mathrm{meV}$. In the case of $d_{s}=0 \mathrm{~nm}$, excellent agreement is found using two resonances: a symmetric (antisymmetric) mode at $E_{s}=1.93 \mathrm{eV}$ $\left(E_{a}=1.38 \mathrm{eV}\right)$ with $\Gamma_{s}=380 \mathrm{meV}\left(\Gamma_{a}=60 \mathrm{meV}\right)$ and $\phi_{s}=0$ $\left(\phi_{a}=0.98 \pi\right)$. This quantitative analysis provides important information on the coupling mechanism. For example, a lattice displacement of $d_{s}=100 \mathrm{~nm}$ affects the spectral positions of both resonances, and $E_{s}\left(E_{a}\right)$ is shifted to $1.6 \mathrm{eV}$ $(1.76 \mathrm{eV})$. Furthermore, while the linewidth of the subradiant mode $\Gamma_{a}$ is not modified, $\Gamma_{s}$ is increased to $450 \mathrm{meV}$.

The physical origin of the spectral shifts can be understood when regarding the restoring forces induced by the collectively displaced nanowire conduction band electrons. For example, the appearing restoring forces in a structure with $d_{s}=0 \mathrm{~nm}$ are reduced (increased) in case of the antisymmetric (symmetric) mode. The surface charge distribution at the upper wire is facing the opposite (identical) charge distribution of the lower wire. This fact is continuously changed when the displacement is increased to $d_{s}=100 \mathrm{~nm}$. Now the oscillating charges at the upper wire perceive the arising surface charges at the edges of both neighboring wires of the lower lattice plane. Effectively, the restoring forces are altered such that symmetric and antisymmetric modes change their $L_{s p}$-dependent frequency characteristics.

As expected, the induced spectral shifts are characterized by a strong spacer layer thickness dependence. The dispersions of the antisymmetric surface plasmon modes, extracted from our numerical calculations, are displayed in Fig. 3(c). These curves indicate that already a spacer thickness of approximately $L_{s p}=100 \mathrm{~nm}$ is sufficient to avoid the near-field induced $d_{s}$ dependence.

A more complete picture of the particle plasmon hybridization scheme is gained from Fig. 4. The calculated reflection and absorption contour maps are shown in dependence on the vertical shift $d_{s}$. The calculations impressively reveal that the spacer-dependent interlayer coupling can indeed be classified into two regimes. Radiative coupling is the only interaction mechanism for structures with $L_{s p}>100 \mathrm{~nm}$ (farfield regime), while spectra for smaller separations show an additional dependence on strong near-field components (near-field regime). In particular, the spectral response is not
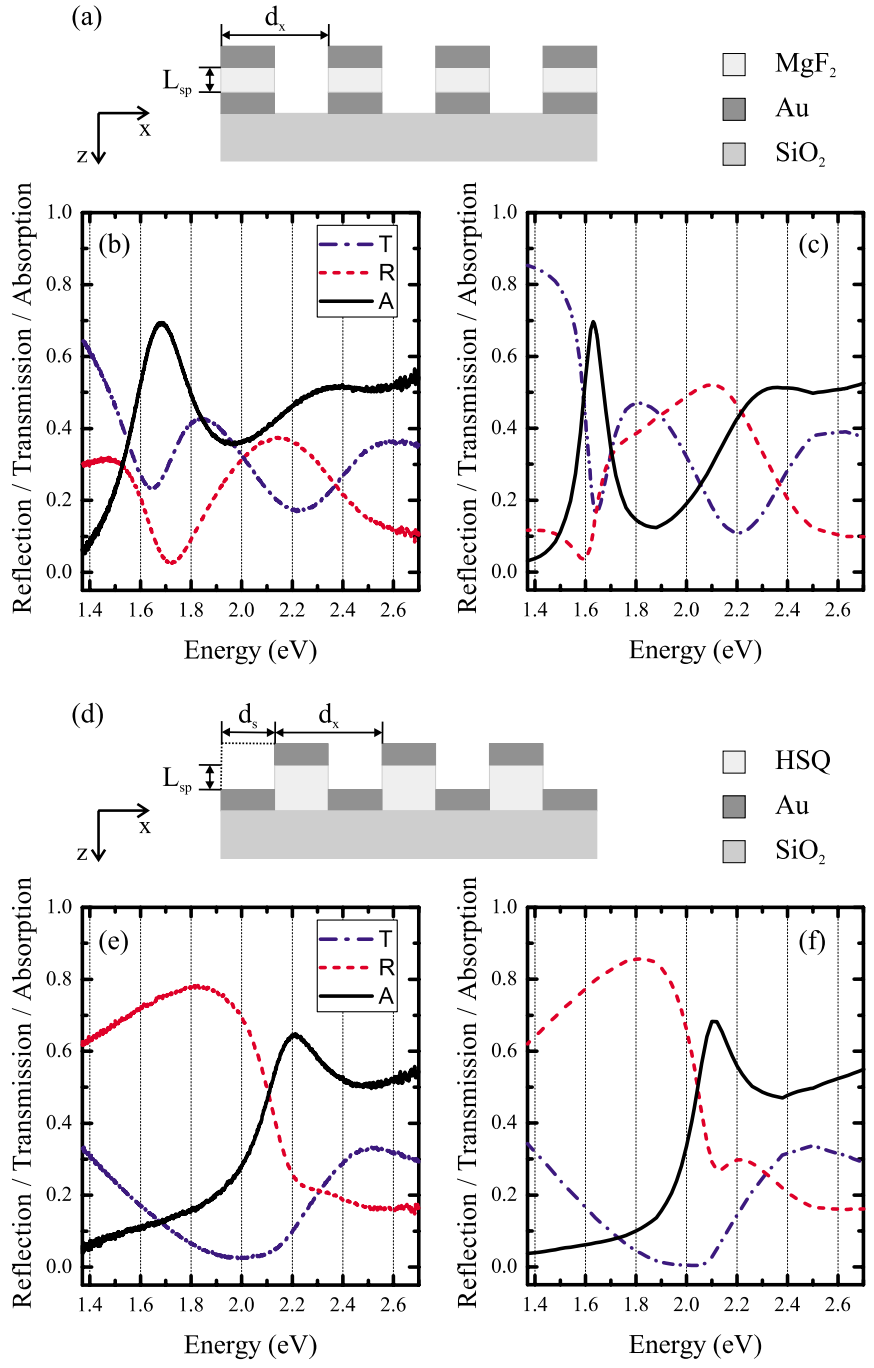

FIG. 5. (Color online) Reflection $(R)$, transmission $(T)$, and absorption $(A)$ spectra for lattice geometries with $d_{x}=200 \mathrm{~nm}$. The experimental spectra in (b) for a structure with $d_{s}=0 \mathrm{~nm}$ and $L_{s p}$ $=25 \mathrm{~nm}$ are compared with corresponding numerical results in (c). The experimental and numerical spectra for lattices with $d_{s}$ $=100 \mathrm{~nm}$ and $L_{s p}=17 \mathrm{~nm}$ are shown in (e) and (f).

sensitive to the exact lattice alignment in the far-field regime for $L_{s p}>100 \mathrm{~nm}$ : identical reflection and absorption spectra are obtained for arbitrary displacements $d_{s}$ when $L_{s p}$ $>100 \mathrm{~nm}$. Pointing out that Bragg-spaced lattices and resonant band gap formation will be separately addressed in a forthcoming presentation, we restrict ourselves to the discussion of the near-field regime. The commonly discussed plasmon hybridization into symmetric and antisymmetric modes is illustrated in Fig. 4(a). The reflection maximum of the symmetric mode at higher energies is continuously increased in energy until the limit of touching wires is reached. In contrast to the symmetric mode, the subradiant antisymmetric mode at lower energies can be traced in the reflection as well as in the corresponding absorption maps. Starting from approximately $1.5 \mathrm{eV}$, the spectral width and the photon energy are reduced when the spacer thickness is decreased. It is important to remark that the energy splitting of sub- and superradiant modes should vanish in a system without near- 
field interaction when $L_{s p}$ approaches zero. Similar to Braggspaced lattice planes, radiative interaction would then result in two degenerate surface plasmon modes: a superradiant mode characterized by effective coupling to the radiation field, and an optically inactive subradiant mode. Surprisingly, the displacement of the lattices induces a complete inversion of the plasmon hybridization scheme. As demonstrated in Figs. 4(b)-4(e), the antisymmetric eigenmode starts to bend upwards while decreasing the spacer thickness. The complete inversion is found for a maximum shift of $d_{s}=100 \mathrm{~nm}$ in Fig. 4(f). Now the crossing of the symmetric and antisymmetric surface plasmon modes results in a Fano interference and hence a nearly complete suppression of the reflection within a narrow band.

\section{EXPERIMENTAL VERIFICATION}

Experimental results show excellent agreement with our numerical predictions. For example, measured reflection, transmission, and absorption $(A=1-R-T)$ spectra are shown in Fig. 5 for two characteristic lattice geometries. Extreme ultraviolet (euv) interference lithography has been used to fabricate large area $\left(1 \times 1 \mathrm{~mm}^{2}\right)$ plasmonic lattices with periods of $d_{x}=200 \mathrm{~nm}$ and wire cross sections of 100 $\times 20 \mathrm{~nm}^{2}$. The first example is a double-layer structure with $d_{s}=0 \mathrm{~nm}$ and $L_{s p}=25 \mathrm{~nm}$. As shown in Fig. 5(a), the wire pairs have been fabricated by evaporating $\mathrm{Au}$ and $\mathrm{MgF}_{2}$ layers before the final lift-off step. The lattice $\left(d_{s}=100 \mathrm{~nm}\right)$ in Fig. 5(d) is simply based on the structured hydrogen silsesquioxane (HSQ) photoresist layer $\left(\varepsilon_{\mathrm{HSQ}}=1.69\right)$ as an appropriate spacer $\left(L_{s p}=25 \mathrm{~nm}\right)$. Thus, only a single evaporation step without lift-off is required.

The spectra in Fig. 5 clearly reveal the expected spectral modifications, although the geometry of the investigated structures is less symmetric (i.e., no $\mathrm{SiO}_{2}$ coverage of the grating). In particular, the near-field-induced inversion of the plasmon hybridization can be directly observed. In Figs. 5(b) and $5(\mathrm{c})$, the subradiant mode $(\approx 1.65 \mathrm{eV})$ is redshifted in comparison to the superradiant mode $(\approx 2.1 \mathrm{eV})$. As displayed in Figs. 5(e) and 5(f), the spectral position of the subradiant mode is increased in energy $(\approx 2.1 \mathrm{eV})$ and is now located above the superradiant mode. Note that inhomogeneous broadening affects the Fano features in the experimental spectra. Particularly, the reflection spectrum in Fig. 5(b) shows slight deviations close to the position of the subradiant mode. This observation might be related to the fact that the exact shape of the upper nanowire slightly deviates from the lower one. Future experimental work has to be focused on the fabrication of plasmonic lattices with asymmetric wire alignments and small spacer thicknesses, which requires the use of planarization techniques.

\section{CONCLUSION}

In conclusion, we have studied the influence of near-field interactions in a radiatively coupled plasmonic lattice. It has been demonstrated that plasmon hybridization can be reversed by directly controlling the near-field-induced coupling. In particular, the spectral interference between superand subradiant modes can be altered by simply modifying the symmetry of the lattice. The discussed fundamental phenomena will allow a broad range of applications, including the design of novel metamaterials, filters, and sensors.

\section{ACKNOWLEDGMENTS}

We acknowledge support from the European Commission (Grant No. FP6-202-IST-1-507879), the Swiss National Science Foundation (Contract No. R'Equip 206021), the Russian Academy of Sciences, and the Russian Foundation for Basic Research (Grant No. 06-02-17211). Part of this work was performed at the Swiss Light Source, Paul Scherrer Institut, Villigen, Switzerland. It is a pleasure to acknowledge stimulating discussions with S. A. Ramakrishna.

*andre.christ@epfl.ch

${ }^{1}$ R. H. Dicke, Phys. Rev. 93, 99 (1954).

${ }^{2}$ I. H. Deutsch, R. J. C. Spreeuw, S. L. Rolston, and W. D. Phillips, Phys. Rev. A 52, 1394 (1995).

${ }^{3}$ E. L. Ivchenko, A. Nesvizhskii, and S. Jorda, Phys. Solid State 36, 1156 (1994).

${ }^{4}$ M. Hübner, J. Kuhl, T. Stroucken, A. Knorr, S. W. Koch, R. Hey, and K. Ploog, Phys. Rev. Lett. 76, 4199 (1996).

${ }^{5}$ U. Kreibig and M. Vollmer, Optical Properties of Metal Clusters (Springer-Verlag, Berlin, 1995).

${ }^{6}$ W. L. Barnes, A. Dereux, and T. W. Ebbesen, Nature (London) 424, 824 (2003).

${ }^{7}$ C. Ropers, D. J. Park, G. Stibenz, G. Steinmeyer, J. Kim, D. S. Kim, and C. Lienau, Phys. Rev. Lett. 94, 113901 (2005).

${ }^{8}$ V. Podolskiy, A. Sarychev, and V. Shalaev, J. Nonlinear Opt. Phys. Mater. 11, 65 (2002).

${ }^{9}$ G. Dolling, C. Enkrich, M. Wegener, J. F. Zhou, C. M. Soukoulis, and S. Linden, Opt. Lett. 30, 3198 (2005).

${ }^{10}$ S. Linden, M. Decker, and M. Wegener, Phys. Rev. Lett. 97,

083902 (2006)

${ }^{11}$ F. Garwe, C. Rockstuhl, C. Etrich, U. Hübner, U. Bauerschäfer, F. Setzpfandt, M. Augustin, T. Pertsch, A. Tünnermann, and F. Lederer, Appl. Phys. B: Lasers Opt. 84, 139 (2006).

${ }^{12}$ P. Mühlschlegel, H.-J. Eisler, O. J. F. Martin, B. Hecht, and D. W. Pohl, Science 308, 1607 (2005).

${ }^{13}$ P. Nordlander, C. Oubre, E. Prodan, K. Li, and M. I. Stockman, Nano Lett. 4, 899 (2004).

${ }^{14}$ S. G. Tikhodeev, A. L. Yablonskii, E. A. Muljarov, N. A. Gippius, and T. Ishihara, Phys. Rev. B 66, 045102 (2002).

${ }^{15}$ A. Christ, S. G. Tikhodeev, N. A. Gippius, J. Kuhl, and H. Giessen, Phys. Rev. Lett. 91, 183901 (2003).

${ }^{16}$ A. Christ, T. Zentgraf, S. G. Tikhodeev, N. A. Gippius, J. Kuhl, and H. Giessen, Phys. Rev. B 74, 155435 (2006).

${ }^{17}$ P. B. Johnson and R. W. Christy, Phys. Rev. B 6, 4370 (1972).

${ }^{18}$ U. Fano, Phys. Rev. 124, 1866 (1961).

${ }^{19}$ S. Fan and J. D. Joannopoulos, Phys. Rev. B 65, 235112 (2002).

${ }^{20}$ N. A. Gippius, S. G. Tikhodeev, and T. Ishihara, Phys. Rev. B 72, 045138 (2005). 